\section{Molecular targets for antifibrotic therapy in liver disease: using magic bullets for crossfire rather than a one-sided shotgun attack}

\section{Claus Hellerbrand}

The c-Jun-N-terminal kinase (JNK) is a member of the mitogen-activated protein kinase (MAPK) family. Upon activation, JNK regulates various cellular responses, such as differentiation, proliferation, migration, the immune reaction and cell death. ${ }^{1} \mathrm{JNK}$ activation is also involved in multiple pathways in liver physiology and disease pathogenesis. ${ }^{2}$

Correspondence to Professor Claus Hellerbrand, Department of Internal Medicine I, University Hospital Regensburg, Regensburg D-93053, Germany; claus.hellerbrand@ukr.de
There are three isoforms of JNK in mammals. Whereas JNK3 is mainly expressed in the brain, heart and testis, JNK1 and JNK2 are expressed in most cells, including hepatic parenchymal and non-parenchymal cells. JNK1 and JNK2 have typically been considered to have overlapping or redundant functions. However, it is becoming more and more evident that the JNK isoforms exhibit diverse effects in various cell types, depending on the magnitude and duration of activation. ${ }^{1}$

Hepatic fibrosis is a complex and tightly regulated process that involves various cell types and pathomechanisms. It can be considered as a dysregulated wound healing response to chronic hepatic injury. Activated hepatic stellate cells (HSCs) are the primary effector cells responsible for the extensive extracellular matrix accumulation and scar formation. However, the situation is more complex, as several additional cell types, including parenchymal, non-parenchymal, immune infiltrating and bone marrow cells, play an important role in both the pathogenesis and resolution of liver fibrosis. ${ }^{3}$ Furthermore, hepatocellular proliferation and apoptosis are critical for tissue regeneration and repair, but when dysregulated, these processes contribute to the malignant transformation of hepatocytes and the development of hepatocellular carcinoma (HCC). ${ }^{34}$

Therefore, it is critical to understand the function of distinct JNK isoforms during the various phases and in the various cell types involved in hepatic fibrosis. Examining $j n k 1$ and $j n k 2$ knockout mice has helped tease out the separate functions of these two kinases, and Cre/lox-P 
methods for cell-specific ablation and bone marrow chimaeras have enabled the analysis of JNKs in distinct cell types and organs. Using these approaches, Zhao et $a l^{5}$ significantly expanded our knowledge on the role of Jnk1 in liver fibrosis and HSCs. This work builds upon a previous study by Kluwe et al, ${ }^{6}$ who had reported that hepatic fibrosis decreased in jnk1 deficient mice $\left(\mathrm{JNK} 1^{-/}\right)$but was unchanged or even increased in the absence of $j n k 2\left(\mathrm{JNK} 2^{--}\right)$in two models of hepatic injury, bile duct ligation or carbon tetrachloride administration. Using the same two fibrosis models, Zhao et al analysed mice with a hepatocyte-specific ablation of Jnk1 in addition to JNK $1^{-/-}$ mice. Unexpectedly, the deletion of Jnk1 in hepatocytes had no major impact on the progression of liver disease, whereas both models clearly indicated that Jnk1 in nonhepatocytes was majorly relevant. ${ }^{5}$ Furthermore, bone marrow transplantation experiments excluded bone marrowderived cells from playing a major role in the JNK1-dependent effect on fibrogenesis. ${ }^{5}$ In contrast, and similar to the results observed by Kluwe et al, ${ }^{6} \mathrm{JNK}$ was strongly activated in myofibroblasts following the induction of liver fibrosis in murine livers, as well as in human fibrotic livers. ${ }^{5}$ The in vitro deletion of Jnk1 in HSCs reduced the lifespan and decreased the differentiation of HSCs into matrix-producing myofibroblasts, ${ }^{5}$ indicating that Jnk1 activation mediates the transactivation and inhibits the apoptosis of HSCs. Together, these data suggest that JNK1 in HSCs is important for fibrosis progression in both murine models. Strikingly, acute and chronic hepatocellular injury was also significantly lower in $\mathrm{JNK} 1^{-/-}$but not in mice with a hepatocyte-specific JNK-1 ablation, ${ }^{5}$ also indicating that JNK1 in hepatocytes fails to play a role in these pathological effects. In concordance with these data, another previous study indicated that isolated JNK1 deficiency, as well as compound JNK1 and JNK2 deficiency, reduced hepatic inflammation and hepatocancerogenesis following exposure to the carcinogen diethylnitrosamine (DEN) in mice. ${ }^{7}$ In contrast, compound JNK deficiency in hepatocytes does not inhibit HCC but indeed increased the tumour burden in the DEN model. ${ }^{7}$ This puzzling finding indicates that the carcinogenic effects of JNK might be restricted to non-parenchymal cells rather than hepatocytes, in which it even functions to reduce tumour development. One can only speculate which non-parenchymal cells are responsible for the anti-HCC effect, but given the known role of activated HSCs in
HCC development and progression, ${ }^{4}$ their contribution appears likely.

Despite the important findings presented by Zhao et al, ${ }^{5}$ their study leaves some open questions. Therefore, the general isoform-specific functions of JNK2 in HSC and hepatic fibrosis remain unclear. However, the study by Kluwe et $a l^{6}$ raises an alarm regarding indiscriminate JNK inhibition and-together with the present study by Zhao et $a l^{5}$ - underscores that the selective inhibition of the JNK1 isoform could offer an effective treatment for hepatic fibrosis.

A therapeutic approach of global JNK inhibition may be even more problematic in non-alcoholic fatty liver disease (NAFLD), in which the distinct functions of JNK1 and JNK2 have been clearly delineated. ${ }^{8}$ Thus, Jnk2-deficient mice placed on a high-fat diet (HFD) presented a similar degree of hepatic steatosis as wild-type mice, but had more severe hepatocellular injury, more advanced obesity and insulin resistance. In contrast, HFD-induced hepatocyte injury and steatosis were suppressed in Jnk1-deficient mice. However, the hepatocyte-specific ablation of jnk1 does not protect mice from HFD-induced insulin resistance, but on normal diets, these mice exhibit increased lipogenesis, attenuated insulin resistance and the spontaneous development of hepatic steatosis. ${ }^{8}$ On the one hand, these findings indicate an anti-steatotic and anti-diabetic function of JNK1 in hepatocytes. On the other hand, JNK1 functions in non-parenchymal liver cells or extrahepatic tissues appear to be critical mediators of hepatocellular steatosis and injury in fatty liver disease. In fact, selective JNK1 deletion in haematopoietic cells prevented hepatic inflammation and the fibrosis induced by a HFD or a cholinedeficient L-amino acid-defined diet, respectively, in mice. Therefore, strong evidence concludes that JNK1 in (hepatic) macrophages (Kupffer cells) contributes to the fibrotic response in NAFLD. Furthermore, studies indicate that the effects of JNK1 in adipocytes and cells of the nervous system promote the development of the metabolic syndrome and the progression of NAFLD, whereas JNK1 in muscle cells might prevent these pathologies. ${ }^{8}$

These findings of JNK functions in NAFLD teach us that for the treatment of liver fibrosis, specific cells and defined molecular pathways must be precisely targeted because even within the same cell type, different isoforms of the same MAPK may exhibit completely opposite effects. Similarly, transcription factor nuclear factor- $\kappa \mathrm{B}(\mathrm{NF}-\kappa \mathrm{B})$, another master regulator of hepatocellular injury, liver fibrosis and
HCC, has been shown to act as a two-edged sword, and the inhibition of distinct components of the NF- $\kappa \mathrm{B}$ signalling cascade may also negatively impact hepatic injury and fibrosis. ${ }^{9}$ Further examples include cytokines, such as interferon-gamma (IFN- $\gamma$ ), transforming growth factor $\beta 1$ (TGF- $\beta$ 1) and Interleukin-10 (IL-10), that have been shown to have pleiotropic activities with effects in one cell type being counterbalanced by opposite effects in a neighbouring cell type. ${ }^{10}$ Therefore, systemic inhibition of such cytokines or broad intervention into signalling pathways may have mixed effects on hepatic fibrogenesis or may even be detrimental, such as in carcinogenesis.

Over the past few years, homing devices on each and every hepatic cell type have been developed. ${ }^{10}$ For example, antibodies and peptides binding to the platelet-derived growth factor receptor or synaptophysin receptor, or coupling mannose-6-phosphate (M6P) to albumin to create a molecule that binds to the M6P/insulin-like growth factor II receptor, provide an important step towards a HSC-specific treatment. ${ }^{10}$ These magic bullets can be used to target defined molecular pathways in specific cells to treat liver fibrosis in patients with chronic liver disease. The study by Zhao et al ${ }^{5}$ provides the molecular basis to consider HSC-specific Jnk1 inhibition as a promising therapeutic strategy. However, the optimal targets or cells may vary depending on the type of liver disease, phase of hepatic injury or specific patient characteristics. This problem will require the application of magic bullets with various types of cell specificity and molecular targets. In addition, combinations of multiple magic bullets may be used to hit fibrosis in the crossfire from varied directions, whereas shotgun attacks from only one side do not appear useful.

\section{Competing interests None.}

Provenance and peer review Commissioned; internally peer reviewed.

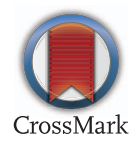

To cite Hellerbrand C. Gut 2014;63:1039-1041.

Received 26 September 2013

Accepted 27 September 2013

Published Online First 16 October 2013

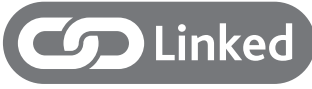

- http://dx.doi.org/10.1136/gutjnl-2013-305507

Gut 2014;63:1039-1041

doi:10.1136/gutjnl-2013-305908 


\section{REFERENCES}

1 Weston CR, Davis RJ. The JNK signal transduction pathway. Curr Opin Cell Biol 2007;19:142-9.

2 Seki E, Brenner DA, Karin M. A liver full of JNK: signaling in regulation of cell function and disease pathogenesis, and clinical approaches. Gastroenterology 2012;143:307-20.

3 Liu X, Xu J, Brenner DA, et al. Reversibility of Liver Fibrosis and Inactivation of Fibrogenic Myofibroblasts. Curr Pathobiol Rep 2013;1:209-14.

4 Kocabayoglu P, Friedman SL. Cellular basis of hepatic fibrosis and its role in inflammation and cancer. Front Biosci (Schol Ed) 2013;5: 217-30.

5 Zhao G, Hatting M, Nevzorova YA, et al. Jnk1 in murine Hepatic Stellate Cells is a crucial mediator of liver fibrogenesis. Gut 2014;63: 1159-72.

6 Kluwe J, Pradere JP, Gwak GY, et al. Modulation of hepatic fibrosis by c-Jun-N-terminal kinase inhibition. Gastroenterology 2010;138:347-59.

7 Das M, Garlick DS, Greiner DL, et al. The role of JNK in the development of hepatocellular carcinoma. Genes Dev 2011;25:634-45.
8 Czaja MJ. JNK regulation of hepatic manifestations of the metabolic syndrome. Trends Endocrinol Metab 2010:21:707-13.

9 Luedde T, Schwabe RF. NF- $\kappa B$ in the liver-linking injury, fibrosis and hepatocellular carcinoma. Nat Rev Gastroenterol Hepatol 2011;8:108-18.

10 Poelstra K, Beljaars L, Melgert BN. Cell-specific delivery of biologicals: problems, pitfalls and possibilities of antifibrotic compounds in the liver. Drug Discov Today Published Online First: 31 May 2013. pii: S1359-6446(13)00159-1. doi:10.1016/j. drudis.2013.05.013 


\section{GUT}

Molecular targets for antifibrotic therapy in liver disease: using magic bullets for crossfire rather than a one-sided shotgun attack

Claus Hellerbrand

Gut 2014 63: 1039-1041 originally published online October 16, 2013 doi: 10.1136/gutjnl-2013-305908

Updated information and services can be found at:

http://gut.bmj.com/content/63/7/1039

These include:

References This article cites 8 articles, 1 of which you can access for free at: http://gut.bmj.com/content/63/7/1039\#BIBL

Email alerting

Receive free email alerts when new articles cite this article. Sign up in the service box at the top right corner of the online article.

Notes

To request permissions go to:

http://group.bmj.com/group/rights-licensing/permissions

To order reprints go to:

http://journals.bmj.com/cgi/reprintform

To subscribe to BMJ go to:

http://group.bmj.com/subscribe/ 